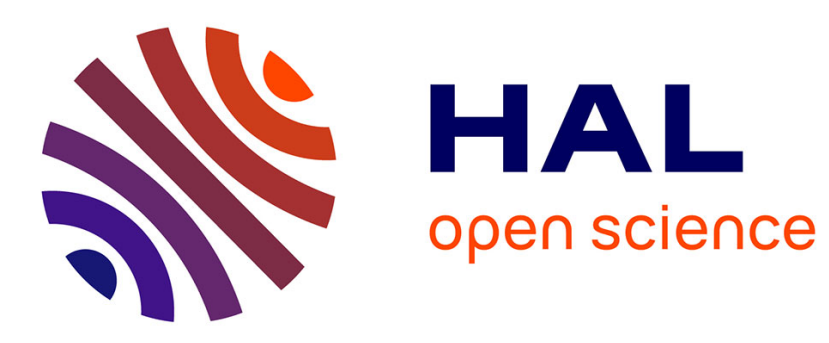

\title{
Plasma Induced Defects in GaAs Probed by a Monoenergetic Positron Beam
}

A. Uedono, Lumei Wei, T. Kawano, S. Tanigawa, K. Wada, H. Nakanishi

\section{To cite this version:}

A. Uedono, Lumei Wei, T. Kawano, S. Tanigawa, K. Wada, et al.. Plasma Induced Defects in GaAs Probed by a Monoenergetic Positron Beam. Journal de Physique IV Proceedings, 1995, 05 (C1), pp.C1-87-C1-90. 10.1051/jp4:1995110 . jpa-00253546

\section{HAL Id: jpa-00253546 https://hal.science/jpa-00253546}

Submitted on 1 Jan 1995

HAL is a multi-disciplinary open access archive for the deposit and dissemination of scientific research documents, whether they are published or not. The documents may come from teaching and research institutions in France or abroad, or from public or private research centers.
L'archive ouverte pluridisciplinaire HAL, est destinée au dépôt et à la diffusion de documents scientifiques de niveau recherche, publiés ou non, émanant des établissements d'enseignement et de recherche français ou étrangers, des laboratoires publics ou privés. 


\title{
Plasma Induced Defects in GaAs Probed by a Monoenergetic Positron Beam
}

\author{
A. Uedono, L. Wei, T. Kawano*, S. Tanigawa, K. Wada** and H. Nakanishi** \\ Institute of Materials Science, University of Tsukuba, Tsukuba, Ibaraki 305, Japan \\ * Radioisotope Center, University of Tsukuba, Tsukuba, Ibaraki 305, Japan \\ ** NTT LSI Laboratories, 3-I Morinosato Wakamiya, Atsugi Kanagawa 243-01, Japan
}

\begin{abstract}
Plasma-induced defects in n-type GaAs were studied by a monoenergetic positron beam. The depth distribution of point-defects was obtained from measurements of Doppler broadening profiles of the annihilation radiation as a function of incident positron energy. From the measurements, it was found that the damaged layer induced by Ar plasma irradiation extended far beyond the stopping range of $\mathrm{Ar}$ ions. The nature of the dominant defects trapping positrons is discussed.
\end{abstract}

\section{INTRODUCTION}

Dry etching processes of semiconductors have attracted attention because of their selectivity and directionality for fabricating LSI structures [1]. During dry etching processes, substrates are subjected to bombardments by electrons, ions and photons, which introduce damage in the surface and the subsurface regions. This damage affects the performance of LSI devices. However, the mechanism of the introduction of defects by dry etching processes is still open to question. Recently, Wada and Nakanishi [2] studied defects introduced by Ar plasma irradiation in GaAs. By using photo-reflectance (PR) spectroscopy [3], they found that compensating centers were introduced by Ar plasma irradiation and that the depth distribution of such defects extended far beyond the stopping range of Ar ions. Monoenergetic positron beams were successfully used to probe defects in the subsurface regions [4]. The advantage of this technique is that the implantation depth of positrons can be adjusted in the region of interest by changing the incident positron energy. Thus, monoenergetic positrons can be used as a nondestructive depth-sensitive probe for point defects. In the present paper, we apply the monoenergetic positron beam technique in order to probe defects introduced by Ar plasma irradiation in GaAs.

The positron annihilation technique is now a powerful method for the study of point defects in materials [5]. When positrons are implanted into condensed matter, they rapidly slow down to thermal energies and finally annihilate with electrons from the surrounding medium into two $511-\mathrm{keV} \gamma$-quanta. A Doppler broadening profile of such annihilation photons provides the momentum distribution of the annihilating electrons. The shape of the Doppler broadening spectrum is characterized by a line-shape parameter $S$ which is defined as the ratio of the central region to the total number of counts in the Doppler broadening energy spectrum. In a material containing defects, a freely diffusing positron may be localized in a vacancy-type defect by the Coulomb repulsion from surrounding ion cores. Since the momentum distribution of electrons in such defects is different from that in the bulk, one can detect defects through measurements of Doppler broadening profiles of the annihilation radiation. In general, the trapping of positrons by vacancy-type defects increases the value of the $S$ parameter. The value of $S$ differs depending on the type of defect; for example, the specific value of the $S$ parameter for a divacancy $\left(V_{2}\right)$ is larger than that for a monovacancy $(V)$. The change in the value of the $S$ parameter due to the annihilation from the trapped state can be observed for vacancy-type defects with a concentration between $\sim 10^{15} \mathrm{~cm}^{-3}$ and $\sim 10^{19} \mathrm{~cm}^{-3}$. 


\section{EXPERIMENT}

The specimen used in the present experiment was a Si-doped GaAs $\left(n=2 \times 10^{17} \mathrm{~cm}^{-3}\right)$ wafer grown by using the Horizontal Bridgman (HB) method. The apparatus for Ar plasma irradiation used in the present experiment was described elsewhere [2]. Before Ar plasma irradiation, the specimen was chemically etched in order to eliminate any existing residual damage on the surface. The etch depth was about $500 \mathrm{~nm}$. Then, the specimen was irradiated by an Ar plasma generated in a parallel plate reactor at a power of $50 \mathrm{~W}$ under a pressure of $10 \mathrm{~Pa}$ for $1 \mathrm{~min}$. The plasma etch depth was about $2 \mathrm{~nm}$. Since the energy of Ar ions was considered to be below $\sim 200 \mathrm{eV}$, the maximum implantation depth of Ar ions was estimated at $1 \sim 2 \mathrm{~nm}$.

The monoenergetic positron beam line was used in order to detect defects introduced by the irradiation [6]. Monoenergetic positrons were moderated from high-energy $(<0.5 \mathrm{MeV})$ positrons emitted from a $22 \mathrm{Na}$ source. The monoenergetic positrons obtained were guided by a magnetic field through multiple discrete acceleration lenses and struck the specimen with an energy adjusted between 0 $\mathrm{keV}$ and $30 \mathrm{keV}$. Doppler broadening profiles of the annihilation radiation were measured by a $\mathrm{Ge}$ detector as a function of incident positron energy. The annihilation spectrum was characterized by the $S$ parameter.

The observed $S-E$ relation was analyzed by the computer code "VEPFIT" developed by van Veen $e t$ al. [7]. The one-dimensional diffusion model of positrons is described by [4]

$$
D+\frac{\mathrm{d}^{2}}{\mathrm{dz}^{2}} n(z)-\kappa_{\mathrm{eff}} n(z)+P(z, E)=0,
$$

where $D_{+}$is the diffusion coefficient of positrons, $n(z)$ is the probability density of positrons at a distance $z$ from the surface, $\kappa_{\text {eff }}$ is the effective escape rate of positrons from the diffusion process and $P(z, E)$ is the implantation profile of positrons. The diffusion length of positrons, $L_{\mathrm{d}}$, is given by

$$
L_{\mathrm{d}}=\sqrt{D_{+} / \kappa_{\mathrm{eff}}} .
$$

In VEPFIT, $P(z, E)$ is described by the following expression:

$$
P(z, E)=\frac{m z z^{m-1}}{z_{0}^{m}} \exp \left[-\left(\frac{z}{z_{0}}\right)^{m}\right],
$$

where $m$ is the shape parameter of $P(z, E) . \quad z_{0}$ is proportional to the mean implantation depth of positrons, $\bar{z}$, as follows; $z_{0}=\bar{z} / \Gamma[(1 / m)+1]$, where $\Gamma$ is the gamma function. $\bar{z}$ has a power-law dependence on the incident positron energy, $\bar{z}=A E^{\mathrm{n}}$, where $A$ is a constant depending on the density of the target, $\rho,(A=C / \rho, C$ is a constant) and $n$ is an energy- and material-dependent constant. In this article, the values of $m, n$ and $C$ were fixed as $2,1.6$ and $3.32 \mu \mathrm{gcm}^{-2} \mathrm{keV}^{-1.6}$, respectively [8]. For the specimen before Ar plasma irradiation, the observed $S-E$ plot was fitted by the relation,

$$
S(E)=S_{\mathrm{S}} F_{\mathrm{S}}(E)+S_{\mathrm{b}} F_{\mathrm{b}}(E),
$$

where $S_{\mathrm{S}}$ and $S_{\mathrm{b}}$ are the characteristic values of the $S$ parameter for the annihilation of positrons at the surface and in the bulk, respectively. $F(E)$ is the fraction of positrons annihilating at each region $\left(\Sigma F_{\mathrm{i}}(E)=1\right)$. For the specimen after the irradiation, the observed $S-E$ plot was fitted by the relation,

$$
S(E)=S_{\mathrm{S}} F_{\mathrm{S}}(E)+S_{\mathrm{d}} F_{\mathrm{d}}(E)+S_{\mathrm{b}} F_{\mathrm{b}}(E),
$$

where $S_{\mathrm{d}}$ is the characteristic value of the $S$ parameter for the annihilation of positrons trapped by defects.

\section{RESULTS AND DISCUSSION}

Figure 1 shows the $S$ parameter as a function of incident positron energy for the n-type HB-GaAs specimens before and after Ar plasma irradiation. The value of $\bar{z}$ was shown below the horizontal axis in the figure, together with $E$. For both specimens, at high $E(\sim 30 \mathrm{keV})$, the value of the $S$ parameter was found to approach a constant value. This indicates that almost all positrons are implanted into the bulk at this energy range. For the specimen before Ar plasma irradiation, the highest value of $S$ was observed at $E \cong 0 \mathrm{keV}$. This is due to the annihilation of positrons at the surface of the specimen. The observed $S-E$ plot for the specimen before Ar plasma irradiation was fitted by eq. (4), where the electric 


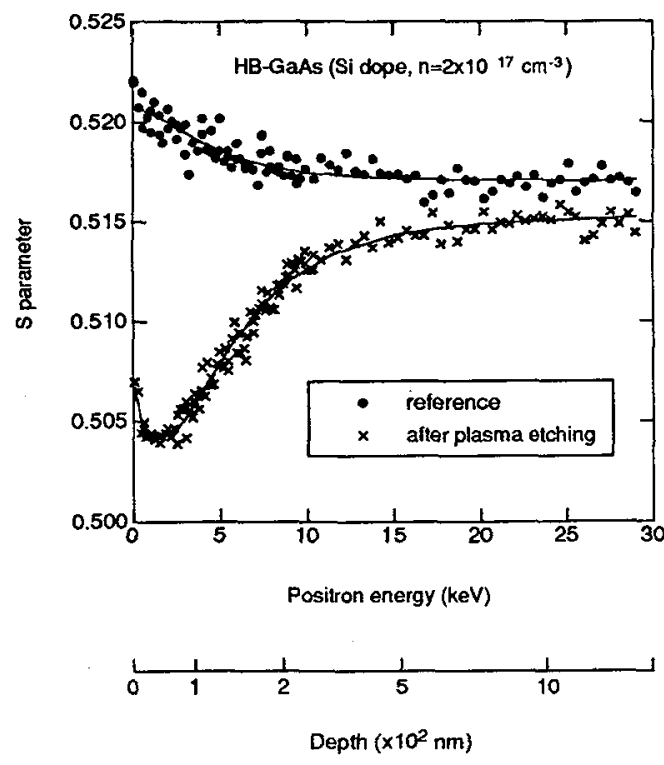

Fig. 1 The lineshape parameter $S$ as a function of incident positron energy for the Si-doped HBGaAs specimens before and after Ar plasma irradiation The solid curves are fits to the experimental data of eq. (4) or (5). field in the specimen was assumed to be negligible. The solid curve shown in Fig. 1 is the result of the fitting. The obtained value of the diffusion length of positrons in the bulk GaAs, $L_{\mathrm{d}(\text { bulk), }}$ and that of $S_{\mathrm{b}}$ are summarized in Table I.

For the specimen after Ar plasma irradiation, the observed $S-E$ plot was fitted by eq. (5). In this fitting, it was assumed that the damaged layer was homogeneous at $0<z<D$ and that all positrons implanted into this region annihilated from the trapped states. The result of the fitting is shown as a solid curve in Fig. 1. The obtained parameters are summarized in Table $I$, where $L_{\mathrm{d}(\mathrm{defect})}$ is the diffusion length of positrons in the damaged layer.

For the specimen before Ar plasma irradiation, the value of $L_{\mathrm{d}}$ (bulk) was found to be smaller than that obtained for an undoped GaAs specimen grown by the liquid-encapsulated Czochralski (LEC) method $(241 \mathrm{~nm})$ [9]. Corbel et al. [10] and Dannefaer et al. [11] already reported that the dominant species of defects in n-type GaAs is monovacancy-type defects. Thus, the observed short diffusion length of positrons for the HBGaAs specimen can be attributed to the trapping of positrons by vacancy-type defects. Uedono $e t$ $a l$. [9] reported that no trapping of positrons was observed in the undoped LEC-GaAs specimen. In order to know the characteristic value of $S$

Table I Summary of fitted parameters for the Si-doped HB-GaAs specimen before and after Ar plasma irradiation.

\begin{tabular}{cccccc}
\hline \hline specimen & $S_{b}$ & $L_{d(b u l k)(\mathrm{nm})}$ & $S_{d}$ & $L_{d(d e f e c t)}(\mathrm{nm})$ & $\bar{D}(\mathrm{~nm})$ \\
\hline before irrad. & $0.5170(1)$ & $86(6)$ & - & - & - \\
after irrad. & $0.5153(1)$ & $113(5)$ & $0.5042(1)$ & $1.4(4)$ & $33(3)$ \\
\hline \hline
\end{tabular}

corresponding to the annihilation of positrons from the delocalized state, the $S-E$ relation for the undoped LEC-GaAs specimen was measured. The value of $S_{\mathrm{b}}$ obtained for this specimen was 0.5143 . The value of $S_{\mathrm{b}}$ obtained for the HB-GaAs specimen before Ar plasma irradiation was larger than that for the undoped LEC-GaAs specimen (Table I). This difference in the value of $S$ can be attributed to the trapping of positrons by monovacancy-type defects in the n-type HB-GaAs specimen.

For the specimen after Ar plasma irradiation, it was found that the damaged region $(D=33 \mathrm{~nm})$ extended far beyond the stopping range of Ar ions (Table I). The depth distribution of compensating centers obtained by PR was in agreement with that obtained in the present experiment [2]. Wei $e t$ al. [12] also reported similar results for a Si specimen after reactive ion etching. They reported that the main species of defects in the damaged region was vacancy-type defects. In the present experiment, however, the value of $S_{\mathrm{d}}$ was smaller than $S_{\mathrm{b}}$ (Table I). Note that $S_{\mathrm{b}}$ is close to the value for the LECGaAs specimen (0.5143). Thus, the main species of defects detected by positron annihilation cannot be identified as vacancy-type defects. Uedono et al. [13] measured the $S$ - $E$ relationship for $200 \mathrm{keV} \mathrm{O} \mathrm{O}^{+}$ ion implanted Si specimens. They found that the characteristic value of $S$ for the annihilation of positrons trapped by defects introduced by $\mathrm{O}^{+}$-ion implantation was smaller than $S_{\mathrm{b}}$. This fact was attributed to the annihilation of positrons trapped by vacancy-oxygen complexes. Because of the broad momentum distribution of the electrons of oxygen atoms, the characteristic value of $S$ for vacancyoxygen complexes is decreased by the annihilation of positrons with electrons of the oxygen atoms. In the present experiment, however, no correlation between $\mathrm{Ar}$ and intrinsic point-defects was expected. Recently, Chadi [14] reported pseudopotential calculations for bonding configurations of selfinterstitials in GaAs. He concluded that $<110>$ split interstitials of $\mathrm{Ga}$ and As could be acceptors with a 
negative $U$ character. Since positrons are considered to be attracted by acceptor-type defects, they could be trapped by negative self-interstitials in GaAs. To explain our results, such trapping will then increase the annihilation probability of positrons with core electrons and consequently decrease the value of $S$. Wada and Nakanishi [2] reported that the diffusivity of compensating centers was higher than that of vacancy-type defects such as Ga-vacancies. Because of the high mobility of such centers, they concluded that the species of defects could be self-interstitials. Their conclusion agrees well with that obtained in the present experiment.

\section{CONCLUSION}

We have studied the defects introduced by At plasma irradiation in the n-type HB-GaAs specimens. Doppler broadening spectra were measured as a function of incident positron energy for the HB-GaAs specimens before and after Ar plasma irradiation. The dominant defect species was identified as interstitial-type defects from the characteristic value of $S$. The depth distribution of the defects was found to extend far beyond the stopping range of $\mathrm{Ar}$ ions. These results are in good agreement with those obtained by PR spectroscopy. The present investigation shows that positrons provide a sensitive and nondestructive probe for point defects introduced by dry etching processes.

\section{REFERENCE}

[1] Schutz R.J., VLSI Technology (McGraw-Hill, New York, 1988) pp. 184-232.

[2] Wada K. and Nakanishi H., Mater. Sci. Forum 143-147 (1994) 1433-1438.

[3] Semiconductor and Semimetals (Academic, New York, 1972).

[4] Schultz P.J. and Lynn K.G., Rev. Mod. Phys. 60 (1988) 701-779.

[5] Positrons in Solids, edited by P. Hautojärvi (Springer-Verlag, Berlin, 1979).

[6] Uedono A., Wei L., Dosho C., Kondo H., Tanigawa S. and Tamura M., Jpn. J. Appl. Phys. 30 (1991) 1597-1603.

[7] Van Veen A., Schut H., de Vries J., Hakvoort R.A. and ljpma M.R., Positron Beams for Solids and Surfaces, AIP Conf. Proc. 218, (AIP, New York, 1990) p. 171-196.

[8] Leung T.C., Asoka-Kumar P., Nielsen B. and Lynn K.G., J. Appl. Phys. 73 (1993) 168-184.

[9] Uedono A., Wei L., Tabuki Y., Kondo H., Tanigawa S., Wada K. and Nakanishi H., Jpn. J. Appl. Phys. 30 (1991) L2002-L2005.

[10] Corbel C., Stucky M., Hautojärvi P., Saarinen K., and Moser P., Phys. Rev. B 38 (1988) 8192-8208.

[11] Dannefaer S., Mascher P. and Kerr D., J. Phys. Condens. Matter 1 (1989) 3213-3328.

[12] Wei L., Tabuki Y. and Tanigawa S., Jpn. J. Appl. Phys. 32 (1993) 7-11.

[13] Uedono A., Wei L., Tanigawa, S. Suzuki R., Ohgaki, H., Mikado T., Kametani H., Akiyama H., Yamaguchi Y. and Koumaru M., Jpn. J. Appl. Phys. 32 (1993) 3682-3689.

[14] Chadi D.J., Phys. Rev. B 46 (1992) 9400-9407. 